\title{
Communications
}

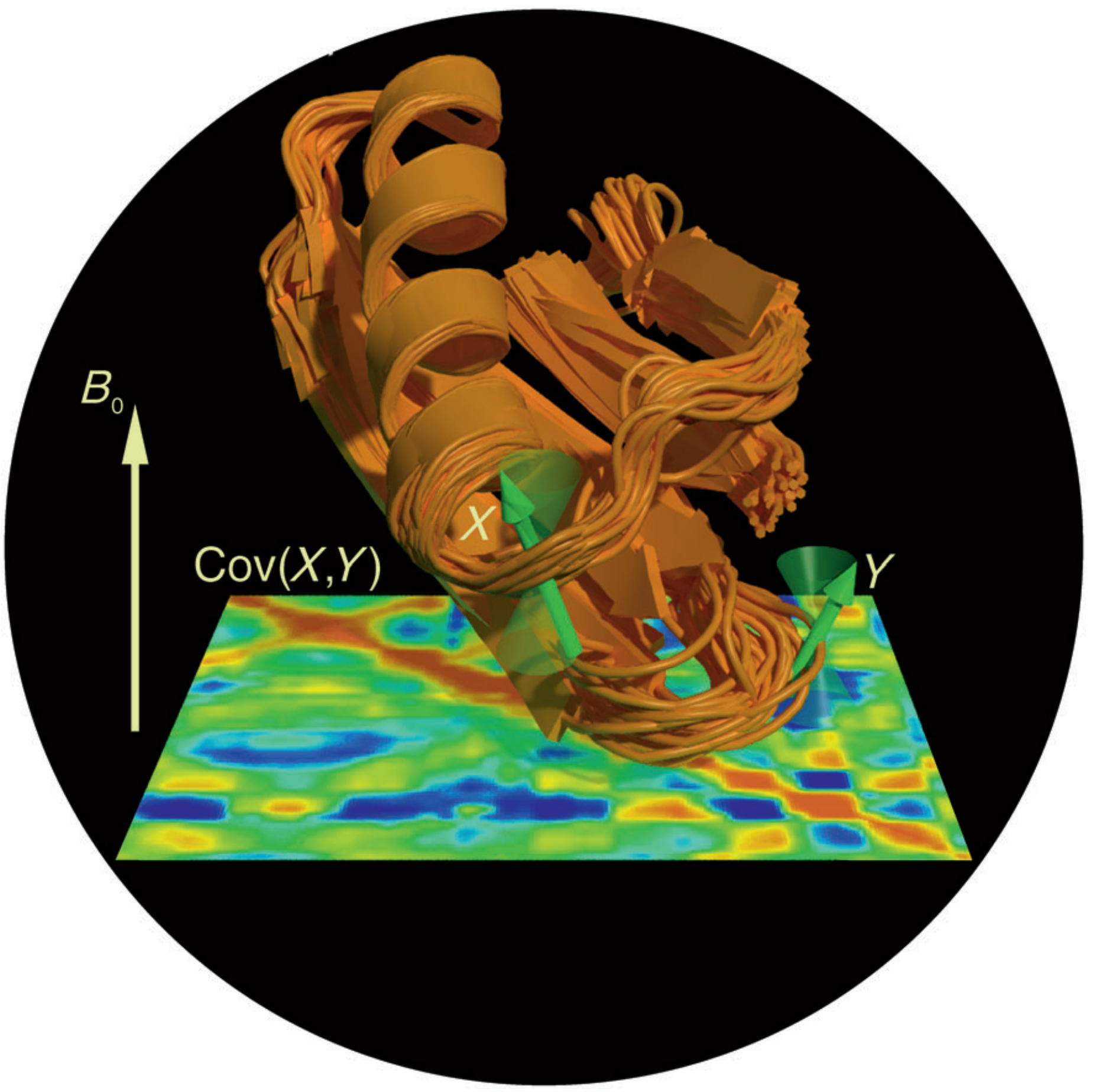

Correlated motions can be accurately extracted from molecular dynamics (MD) simulations that are compatible with the measured NMR spectroscopic data (NOE and order parameters) for two different proteins (Cov: covariance matrix). B. de Groot and co-workers discuss the results of MD simulations on a fragment of protein $G$ in their Communication on the following pages. 


\section{Molecular Dynamics Simulations of Protein G Challenge NMR-Derived Correlated Backbone Motions**}

\author{
Oliver F. Lange, Helmut Grubmüller, and \\ Bert L. de Groot*
}

Protein function is fundamentally linked to underlying structural and molecular dynamics. ${ }^{[1]}$ Many biological functions such as enzymatic activity and ligand binding are controlled or even realized through conformational motions ${ }^{[2]}$ which are typically collective in nature, as is the case with allosteric effects and domain motions. ${ }^{[3]}$ Conformational motions determine energetics, kinetics, and the strength of thermodynamic driving forces through conformational entropy. ${ }^{[4-6]}$

NMR relaxation studies provide a powerful and established method to gain experimental access to fast protein dynamics in atomic detail. ${ }^{[7-9]}$ Model-free analysis of NMR relaxation times, in particular, yields generalized order parameters for individual bond vectors ${ }^{[10]}$ which allows the extraction of information about flexibility and timescales of the motions of individual backbone sites ${ }^{[11]}$ and side chains. ${ }^{[12]}$ Until recently, however, NMR relaxation experiments could only probe the flexibility of individual bond vectors, whereas correlated motions could not be probed. A recently proposed method by Mayer et al. ${ }^{[13]}$ promises to overcome this limitation by measuring the covariation of backbone motion by NMR relaxation studies. Specifically, the authors "propose a general approach to the detection of correlated changes in internal protein motions, which are expected to reflect the underlying influence of correlated dynamics". ${ }^{[13]}$ In this approach, NMR relaxation data for a small domain of protein $\mathrm{G}$ were obtained for 10 mutants at the same sequence position. The resulting perturbations were reported to cause changes in the measured order parameters, which were recorded for each individual residue. For many residue pairs, these changes are significantly correlated (Figure $1 \mathrm{a}$ ), which led the authors to suggest that the observed covariances reflect underlying correlated atomic motions. ${ }^{[13]}$ Whether and how the measured covariations actually reflect correlated atomic motion cannot be resolved by experiment alone, which prevents the direct atomistic interpretation of these types of measurements. To address this issue, we present sub-microsecond molecular dynamics (MD) simulations which,

[*] O. F. Lange, Priv.-Doz. Dr. H. Grubmüller, Dr. B. L. de Groot Theoretical and Computational Biophysics Department Max Planck Institute for Biophysical Chemistry Am Fassberg 11, 37077 Göttingen (Germany) Fax: (+49) 551-2012302 E-mail:degroot@gwdg.de

[**] We thank Christian Griesinger, Sander Nabuurs, and Martin Stone for carefully reading the manuscript.

D) Supporting information for this article is available on the WWW under http://www.angewandte.org or from the author. together with the NMR experimental data, provide a comprehensive picture of the correlated atomic dynamics of the protein $\mathrm{G}$ domain.

We carried out two MD simulations of the B1 domain of protein G, with lengths of 100 and $200 \mathrm{~ns}$, referred to as MD1 and MD2, respectively. Correlations of atomic motions were quantified by correlation matrices calculated from the MD simulations. Figure 1c shows correlation matrices obtained from MD1 (above the diagonal) and MD2 (below the diagonal). The similarity of the two matrices shows that the computed correlations are largely well-converged. Full convergence is not reached for residues $37-42$, which constitute the loop that connects the $\alpha$ helix with the $\beta 3$ strand. Closer inspection revealed long timescale contributions to the loop dynamics. However, the relevant sub-microsecond timescale probed by NMR relaxation experiments ${ }^{[14]}$ is well-sampled in our simulations.

The matrix is dominated by strong correlations between neighboring residues, and appears as a band along the diagonal. The broad region of high correlation between residues 22 and 38 is caused by the 1-4 contacts of the central $\alpha$ helix. The two bands of high correlation perpendicular to the diagonal result from the contacts between different strands of the four-stranded $\beta$ sheet. Strong correlations and anticorrelations are also found between residues $10-15$ of the first hairpin loop and the rest of the molecule. They are caused by a kinking-out motion of the turn between strands $\beta 1$ and $\beta 2$ together with part of the $\beta$ sheet. This motion, hinged around residues 8 and 15, is the main contribution to the principal collective motion (Figure 2), as revealed by principal component analysis. ${ }^{[15,16]}$

To test for a direct connection between the covariation of NMR order parameters and the computed correlated atomic motion observed in the simulation, we compared the computed correlation matrices (Figure $1 \mathrm{c}$ ) with the $S^{2}$ - and the $\tau_{\mathrm{e}^{-}}$ based covariation matrices derived by Mayer et al. ${ }^{[13]}$ (Figure 1a). Overall, the level of correspondence is low. The only feature shared with the MD simulations is the band of anticorrelated motion between residues 10-14, which is present in the experimental results derived from covariations in $\tau_{\mathrm{e}}$ order parameters, but which is not observed in the $S^{2}$ derived correlations. Apart from this detail, the overall lack of similarity is striking. The nature of this discrepancy deserves closer inspection.

An important question is whether the MD simulations describe atomic motions with sufficient accuracy. Potential artifacts include force-field inaccuracies and convergence problems. Therefore, we compared the root mean squared fluctuations (RMSF) of the two MD trajectories (MD1 and MD2) with the RMSF of a structural ensemble of the B1 domain of protein $\mathrm{G}$ obtained from NMR spectroscopic NOE data $^{[17]}$ (PDB entry 3gb1, Experimental Section). The RMSF profiles obtained form the MD simulation agree well with the NOE ensemble (Figure 3). Moreover, the correlation matrices obtained from the simulations (Figure 1c) are in good agreement with that obtained from the NOE ensemble (Figure $1 \mathrm{~b}$ ). These agreements between the simulation- and NOE-derived results are quantified by correlation coefficients of 0.77 and 0.81 for MD1 and MD2, respectively. In 


\section{Communications}

a)

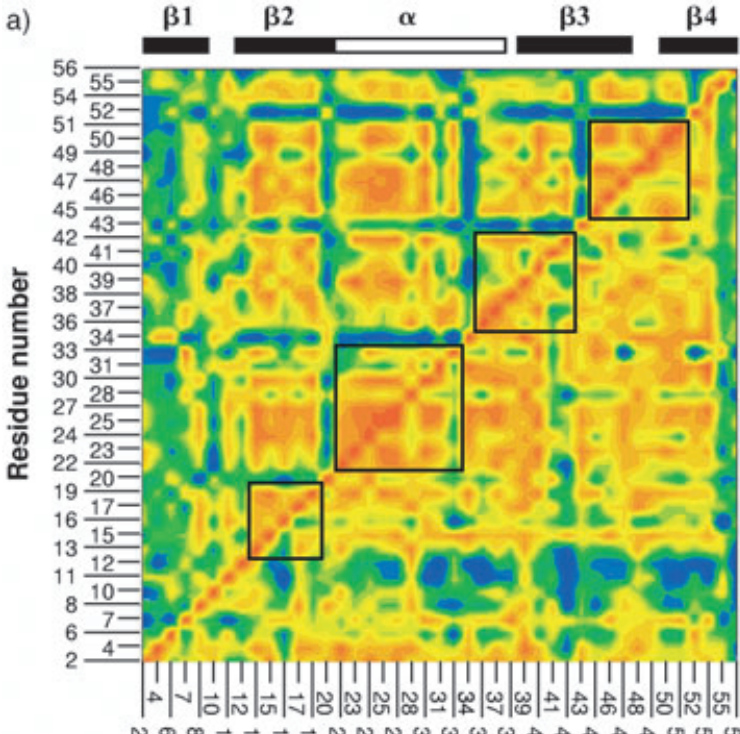

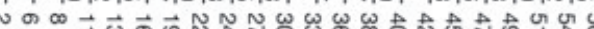

Residue number

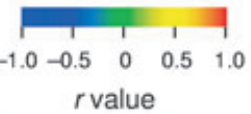

c)

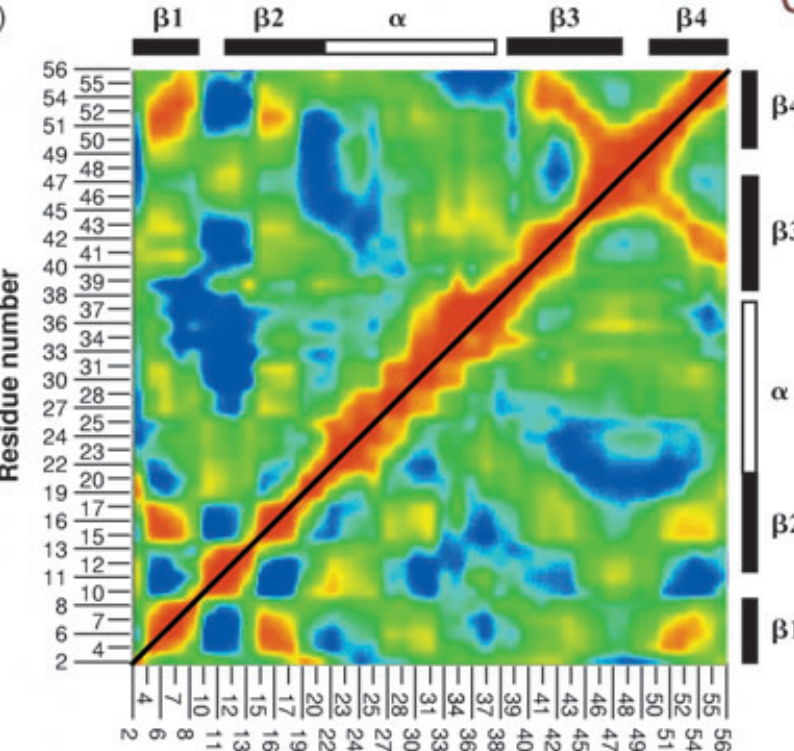

Residue number

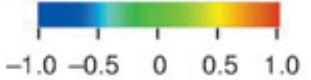

$\beta 4$

$\beta 3$
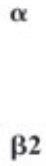

$\beta 1$

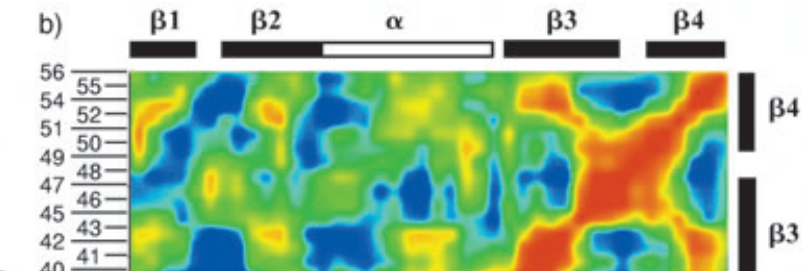

홇

$40 \frac{31}{39-}$
$36 \frac{37}{37}$
$33 \frac{34}{31}$
$30 \frac{28}{27}$
$24 \frac{25}{25}$
$22 \frac{23}{20}$
19

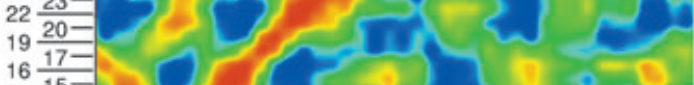

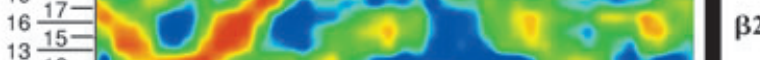

$11 \frac{12-}{10-}$

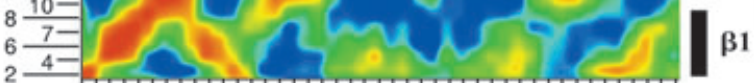

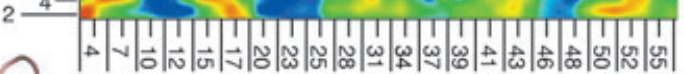

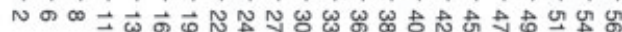

Residue number
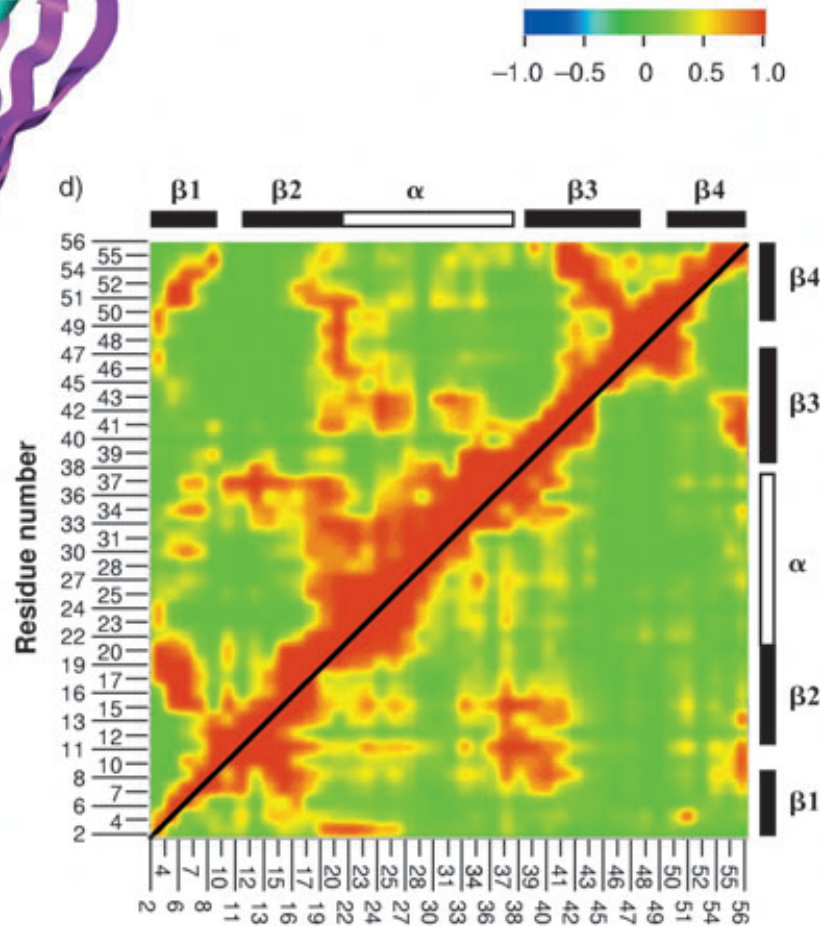

Residue number

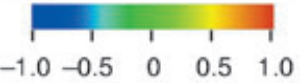

Figure 1. Correlation matrices from NMR experiments and MD calculations. a) Correlation matrices taken with permission from Mayer et al.; ${ }^{[13]}$ data for $S^{2}$ covariations are shown above the diagonal, whereas those for $\tau_{\mathrm{e}}$ covariations are shown below the diagonal (the black boxes are irrelevant for the present discussion). b) Correlation matrix computed from an NMR-NOE structural ensemble. c) Correlation matrices computed from MD simulations. The correlations computed from MD1 are shown above the diagonal; correlations derived from MD2 are below the diagonal. d) Generalized correlations computed from MD2 (above the diagonal); correlations computed for $\mathrm{N}-\mathrm{H}$ vector motions (below the diagonal). The simulated $\mathrm{B} 1$ domain of protein $\mathrm{G}$ is depicted in the center.

contrast, the correlation coefficients between all of these matrices and the covariance matrix computed from order parameters (Figure 1a) are much smaller: 0.06 and 0.04 for the two MD matrices, and 0.05 for the NOE matrices. The good agreement between MD and the NOE ensemble in terms of the atomic fluctuations and particularly their correlations makes it unlikely that the discrepancy with the covariance matrix obtained from order parameters is caused 


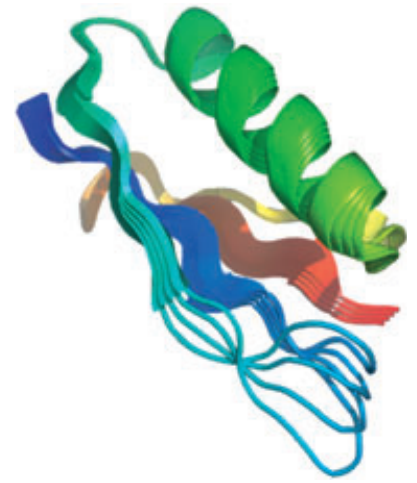

Figure 2. Main collective motion of the $B 1$ domain of protein $G$ as revealed by the principal component analysis described in the text.

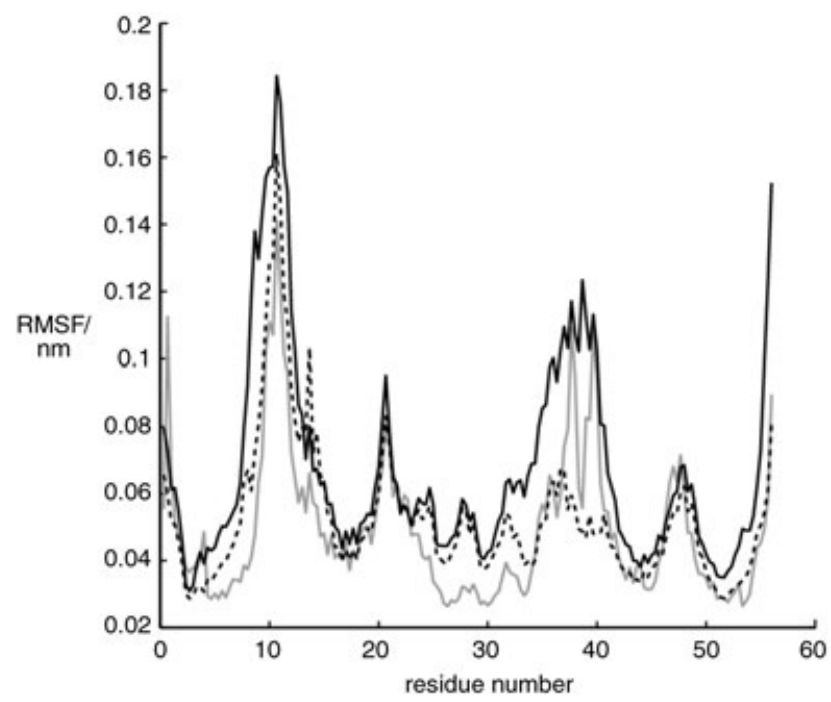

Figure 3. RMSF of backbone atoms observed in the NMR ensemble (gray line), and RMSF computed from the two different MD trajectories (MD1, _ ; MD2, ----): the positions of the major RMSF peaks agree well with the experimental data. The fluctuations of the largest motion (residues 8-15) and of the $\alpha$ helix are slightly overestimated by the MD simulations but overall, the flexibility of the remaining regions of the molecule is well-reproduced.

by simulation artifacts. That this agreement is not just anecdotal is indicated by a correlation coefficient of 0.82 for the NOE and MD covariance matrix obtained for a different protein, ubiquitin (Supporting Information), and by a correlation coefficient of 0.8 obtained with a different NMR data set for protein G (PDB entry 1gb1).

Because order parameters form the basis for the covariances determined by Mayer et al., it is necessary to check if the motions probed by order parameters are accurately described by the MD simulations as well. To this end, we compared the experimentally obtained order parameters ${ }^{[18]}$ for protein $\mathrm{G}$ with those computed from the MD trajectories (Figure 4 a). Within the error bars, the simulations agree with each other as well as with the measured order parameters. The inset of Figure 4 shows that most differences between the observed and computed order parameters are below 0.1 with

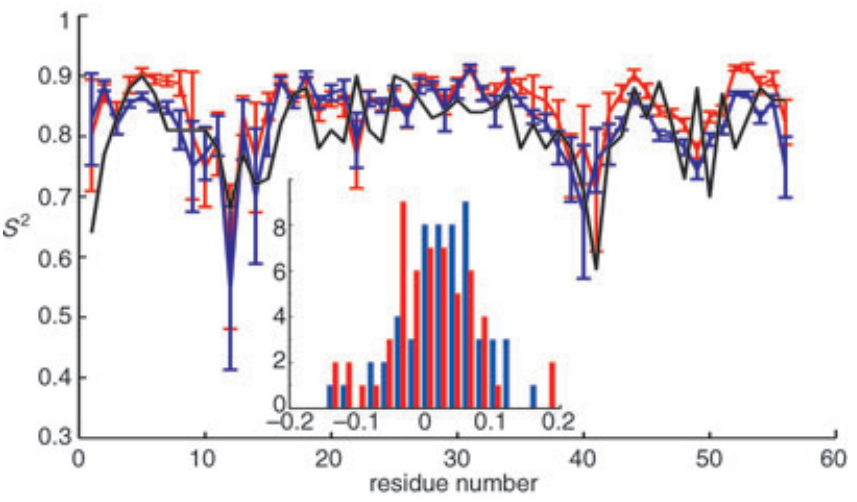

Figure 4. Comparison of the generalized order parameters $\left(S^{2}\right)$ between the MD simulations and the NMR data set. ${ }^{[18]}$ Computed order parameters (in color) with error bars are compared with experimentally observed order parameters (black). Inset: histogram of the order parameter differences between the MD data sets and the experimental results.

few outliers below 0.2. Overall, the agreement is good and is in line with earlier observations. ${ }^{[19-22]}$

We therefore conclude that the simulations provide a comprehensive and accurate picture of the correlated atomic motion within the protein $\mathrm{G}$ domain that is consistent with the available experimental data. The remaining unexplained discrepancy with the covariances derived from NMR experimental order parameters suggests that these two quantities are, in fact, not directly related. Comparison of all elements of the MD correlation matrix with the respective covariations of NMR order parameters as a scatter plot (Figure $5 \mathrm{a}$ ) confirms this finding. Furthermore, the absence of any detectable structure in this plot suggests the absence of any relation to this measure of linearly correlated atomic motion.

At this point, it is important to note that the correlation coefficients as used in the matrices shown in Figures $1 \mathrm{~b}$ and $1 \mathrm{c}$, although widely used, detect only part of all possible correlated atomic motions. For instance, two atoms oscillating with a $\pi / 2$ phase shift will give rise to a vanishing correlation matrix element $\langle\sin (\omega t) \sin (\omega t+\pi / 2)\rangle=0$, and thus, this highly correlated motion would not be detected. This simple example shows that the results discussed so far do not rule out the possibility that the measured covariations of NMR order parameters relate to such "hidden" correlated motions. To detect those as well, we employed a second, more general correlation measure derived from information theory.

To this end, we consider the information $S_{i}$ contained within the motion of each individual atom $i$ as well as the joint information $S_{i, j}$ contained within the (possibly correlated) motion of each pair of atoms $i$ and $j$. As any type of correlation, hidden or not, will decrease $S_{i, j}$ with respect to $S_{i}+S_{j}$, the excess $C_{i, j}=S_{i}+S_{j}-S_{i, j}$ serves as a generalized and very sensitive measure to detect all correlations within atomic motions. We note that an equivalent formulation of the same method would rest on the fact that only for statistically independent data sets $X$ and $Y$, their joint probability is given by $P(X, Y)=P(X) P(Y)$. For the calculation of the information contents from MD trajectories, see the Supporting Information and Ref. [34]. 

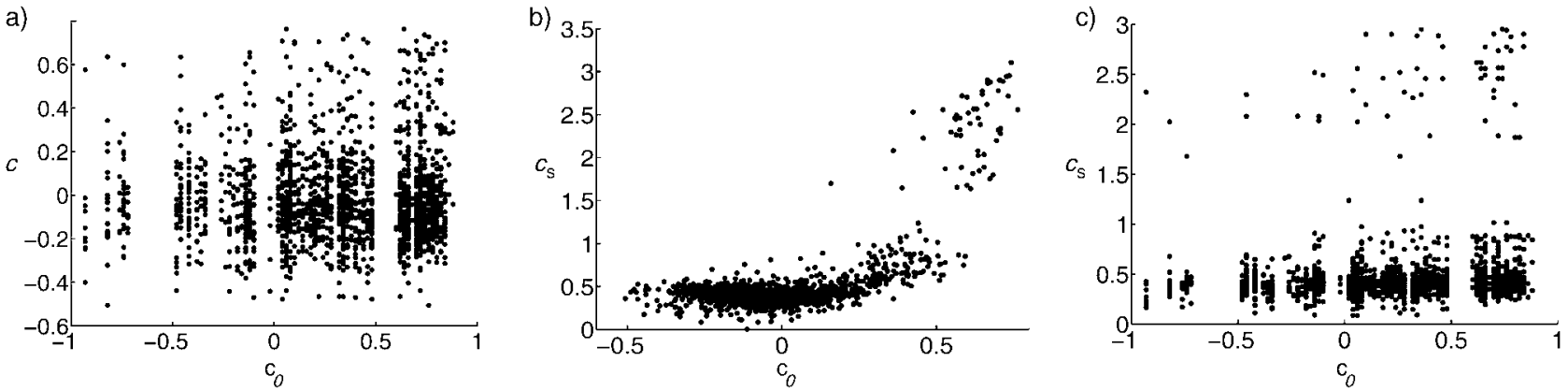

Figure 5. Scatter plots of covariation and correlation matrix elements in which two of the correlation matrices shown in Figure 1 are compared; each point represents the two different correlations obtained with the respective methods for the same residue pair. a) Correlations obtained from MD simulation $c$, as depicted in Figure $1 \mathrm{c}$, are plotted against the covariations of order parameters $c_{0}$, shown in Figure 1 a. b) Generalized correlations $c_{S}$ (Figure $1 \mathrm{~d}$ ) obtained from the MD simulation are plotted against $c_{0}$ (Figure $1 \mathrm{c}$ ), the correlations obtained from MD simulation in the established way. c) Generalized correlations $c_{S}$ (Figure 1d) computed from simulation MD2 plotted against measured covariations of order parameters $c_{0}$ (Figure $1 \mathrm{a}$ ).

Figure 1d (above the diagonal) shows the obtained generalized correlation matrix. Although the overall structure appears similar, minor but significant differences to the linear Pearson correlation matrix (Figure 1c) are observed, which result from the "hidden" correlations now detected by the information theoretical approach. Quantitative comparison of the Pearson matrix elements with the generalized elements (Figure 5b) confirms that the two measures are related (with a correlation coefficient of 0.65 ). In contrast, no such relation is observed to the covariances derived from the NMR order parameters (Figure 5c; correlation coefficient 0.07), which suggests that no significant information on correlated atomic motion in Cartesian space is contained within these NMR spectroscopic data.

It might be argued that a more direct comparison between MD and NMR data would rest on an analysis of the correlation in the orientational fluctuations of $\mathrm{N}-\mathrm{H}$ bond vectors, which are probed by the NMR order parameters, rather than on the Cartesian coordinates. However, these fluctuations are already included within the generalized correlation measure described above and hence, similar results are expected. Figure 1d (below the diagonal) shows that this is indeed the case as quantified by the low correlation coefficient of 0.05 between $\mathrm{N}-\mathrm{H}$ vector fluctuations in $\mathrm{MD}$ and the covariances derived from the NMR order parameters.

In summary, we have demonstrated that for two different proteins, correlated motions can be accurately extracted from MD simulations that are compatible with the measured NMR data (NOE and order parameters). However, the correlated atomic motions described by our sub-microsecond MD simulations of the $\mathrm{B} 1$ domain of protein $\mathrm{G}$ are unrelated to covariations derived from order parameters, even though different measures of correlated motion have been applied. The obtained agreement with independent NMR spectroscopic NOE data and with $\mathrm{N}-\mathrm{H}$ order parameters provides strong evidence that the simulations accurately describe the atomic motions and their correlations at the experimentally relevant timescale. This is further supported by the good qualitative agreement with the results of recently published residual dipolar coupling experiments. ${ }^{[23]}$ Taken together, the results render it unlikely that the observed covariances in measured order parameters reflect the underlying influence of the correlated atomic dynamics for the wild-type protein. Instead, we speculate that the measured covariances are caused by correlated structural changes that result from the introduced point mutations, which in turn, affect the atomic mobilities. In this framework, the experiments would probe remarkably correlated structural plasticities rather than correlated atomic motions. Further simulations of all 10 mutants studied will therefore be required to test this hypothesis and to structurally characterize the properties of this proposed nonlocal plasticity.

\section{Experimental Section}

MD simulations were started from the crystal structure of the B1 domain of streptococcal protein $\mathrm{G}^{[24]}$ (PDB entry 1PGB). Simulations were carried out with the OPLS all-atom force field. ${ }^{[25]}$ The protein was solvated in 4651 TIP4P water molecules. Four sodium ions were added to the simulation system to compensate for the net negative charge of the protein. The total system size comprised 19463 atoms. The simulation was carried out in a cubic box with a minimal distance between solute and box edge of $0.8 \mathrm{~nm}$. Periodic boundary conditions were used. All MD simulations were carried out with the Gromacs simulation suite. ${ }^{[26]}$ LINCS and Settle ${ }^{[27,28]}$ were applied to constrain covalent bond lengths, allowing an integration step of $2 \mathrm{fs}$. Electrostatic interactions were calculated with the particle-mesh Ewald method. ${ }^{[29]}$ The temperature was kept constant by separately coupling ( $\tau=0.1 \mathrm{ps}$ ) the peptide and solvent to an external temperature bath. ${ }^{[30]}$ The pressure was kept constant by weak isotropic coupling ( $\tau=1.0 \mathrm{ps}$ ) to a pressure bath. ${ }^{[30]}$ Simulations were run for $100 \mathrm{~ns}$ (MD1) and $200 \mathrm{~ns}$ (MD2)

An NMR-NOE structure ensemble of 30 structures was generated with the standard simulated annealing protocol in $\left.\mathrm{CNS},{ }^{[31}\right]$ applying the NOE distance bounds as available from the PDB entry 3GB1. ${ }^{[32]}$ In short, individual structures were generated by slow cooling from high-temperature simulations starting from an extended structure and different sets of starting velocities. Each annealing cycle consisted of $15 \mathrm{ps}$ of torsion-angle MD at high temperature $(50000 \mathrm{~K})$ followed by an annealing phase $(15 \mathrm{ps})$ to zero temperature with torsion-angle MD and an annealing phase (15 ps) with Cartesian dynamics from 2000 to $0 \mathrm{~K}$. Finally, each structure was energyminimized by 10 cycles of 200 steepest-decent steps. Default parameters for the scaling of the individual energy terms were used, including the NOE energy term. 
To analyze the data in the molecular coordinate frame, all structures were fitted to the backbone of the crystal structure. RMSF for each ensemble were calculated as $\mathrm{RMSF}_{i}=\sqrt{\left\langle r_{i}^{2}\right\rangle}$, in which $r_{i}$ is the distance of the $i^{\text {th }} \mathrm{C}^{\alpha}$ atom from its average position, and the \langle\rangle denote the average over the whole trajectory with snapshots recorded every picosecond. The correlation matrices were computed as $C_{i, j}=$ $\left\langle\mathbf{x}_{i} \cdot \mathbf{x}_{j}\right\rangle /\left(\left\langle\mathbf{x}_{i}^{2}\right\rangle\left\langle\mathbf{x}_{i}^{2}\right\rangle\right)^{1 / 2}$, in which $\mathbf{x}_{i}$ and $\mathbf{x}_{j}$ are difference vectors between the $i^{\text {th }}$ and $j^{\text {th }} \mathrm{C}^{\alpha}$ atom, respectively, and their average positions in the molecule-fixed frame. To enhance visibility of small differences, the correlations were weighted by the sigmoidal function $W(x)=2 /[1+$ $\exp (-\lambda x)]-1$, with $\lambda=5.5$ before plotting. The enhancement coefficient $\lambda$ was set as strong as possible under the constraint that only statistically significant fluctuations become apparent.

Order parameters $S^{2}$ were defined as the asymptotic value of internal correlation functions. ${ }^{[10]}$ The internal correlation function $C_{\text {int }}(t)$ of the $\mathrm{N}-\mathrm{H}$ bond vector motion is given by $C_{\mathrm{int}}(t)=$ $\left\langle P_{2}(\cos \chi(t))\right\rangle$, where $\chi(t)$ is the angle between the internuclear vectors $r(t)$ and $r(0)$, and $r$ is measured in the molecule-fixed frame. $P_{2}(x)=$ $\left(3 x^{2}-1\right) / 2$ denotes the second Legendre polynomial. Fluctuations in the internuclear separation were not included, because the length of all covalent bonds were fixed by LINCS ${ }^{[27]}$ throughout the simulation. It has been shown previously that the effect of such constraints on order parameters calculated from simulations is negligible. ${ }^{[33]}$

To estimate statistical errors in the obtained order parameters, the MD trajectories were divided into $N$ fragments of length $1 \mathrm{~ns}$ each. For each fragment $s$, internal correlation functions $C_{\text {int }}^{\text {si, }}(t)$ were computed for each bond vector $i$ with snapshots taken every picosecond. Parameters $\chi_{s, i}$ were computed as the average from 480 to $500 \mathrm{ps}$ of the internal correlation function $\mathrm{C}_{\mathrm{int}}^{\mathrm{s}, i}(t)$. The order parameter of bond vector $k$ was computed as the mean of $\chi_{s, i}$ over all the fragments, that is $S_{i}^{2}=\left(\sum_{s}^{N} \chi_{s}^{i}\right) / N$, with the error bars given by:

$\Delta S_{i}^{2}=\frac{1}{\sqrt{N(N-1)}}\left[\sum_{s}^{N} \chi_{s, i}^{2}-\left(\sum_{t}^{N} \chi_{t, i}\right)^{2}\right]^{1 / 2}$

The results do not change significantly if the fragmentation length is changed from $1 \mathrm{~ns}$ to longer time intervals (data not shown).

To obtain the generalized correlation measure $C_{i, j}=S_{i}+S_{i}-S_{i, j}$ between the $\mathrm{C}^{\alpha}$ atoms $i$ and $j$, we computed for all atom pairs $(i, j)$ the information $S_{i}$ of the motion of atom $i$ in its $d=3$ dimensional configurational space as well as the information $S_{i, j}$ of the combined motion of atoms $i$ and $j$ in their $d=6$ dimensional configurational space. Herein, only a sketch of the approach is given; for details, see the Supporting Information and Ref. [34].

Our approach is based on a combination of kernel density estimation and nearest-neighbor estimation. For every snapshot $k$ of the trajectory, an effective neighbor distance $\sigma_{\mathrm{s}}$ is determined by requiring $T=\sum^{N} \mathrm{e}^{\left(\mathbf{x}_{t}-\mathbf{x}_{s}\right)^{2} /\left(2 \sigma_{s}^{2}\right)}$ for a given and fixed $T=20$. In this case, $N$ denotes the number of snapshots, and values of $\mathbf{x}$ represent vectors in the $d$ dimensional configurational space considered. This isotropic averaging over local snapshots typically suffers from highly anisotropic configurational space densities, that is, drastically differing widths of the configurational snapshot ensemble in different directions. Particularly problematic and inaccurate were those directions for which the widths are smaller than $\sigma_{s}$. We therefore treated those $l_{\mathrm{s}}$ narrow directions separately by a Gaussian-derived estimate $S_{\mathrm{G}}=$ $1 / 2 \sum_{h=1}^{l_{s}} \log \lambda_{h}^{s}$, which is particularly suitable for those small-amplitude degrees of freedom of width $\lambda_{h=1 \ldots l_{\mathrm{s}}}^{\mathrm{s}}$, respectively. Taken together, the information was computed by:

$\mathrm{S}=\frac{\log N}{N}-\frac{1}{N} \sum_{s=1}^{N}\left(\log \alpha\left(d-l_{s}\right)-\left(d-l_{s}\right) \log \sigma_{\mathrm{s}}-S_{\mathrm{G}}\right)$

in which $\alpha\left(d_{\mathrm{s}}\right)$ denotes a proportionality factor dependent on the local dimension $d_{\mathrm{s}}=d-l_{s}$, which relates the nearest-neighbor statistics $\sigma_{\mathrm{s}}$ to the information measure $S$. We used 10000 snapshots taken every $20 \mathrm{ps}$ from trajectory MD2 for this analysis.
The correlations in the motion of $\mathrm{N}-\mathrm{H}$ bond vectors were computed by applying the generalized correlation measure to pairs of the normalized internuclear vectors rather than to pairs of atomic positions.

Received: December 16, 2004

Revised: January 28, 2005

Keywords: conformation analysis - correlated motions . molecular dynamics . NMR spectroscopy · proteins

[1] A. J. Wand, Nat. Struct. Biol. 2001, 8, 926.

[2] S. J. Benkovic, S. Hammes-Schiffer, Science 2003, 301, 1196.

[3] P. K. Agarwal, S. R. Billeter, P. T. R. Rajagopalan, S. J. Benkovic, S. Hammes-Schiffer, Proc. Natl. Acad. Sci. USA 2002, 99, 2794.

[4] J. N. Onuchic, Z. Luthey-Schulten, P. G. Wolynes, Annu. Rev. Phys. Chem. 1997, 48, 545.

[5] J. D. Forman-Kay, Nat. Struct. Biol. 1999, 6, 1086.

[6] A. L. Lee, A. J. Wand, Nature 2001, 411, 501.

[7] R. Ishima, D. A. Torchia, Nat. Struct. Biol. 2000, 7, 740.

[8] A. G. Palmer, Annu. Rev. Biophys. Biomol. Struct. 2001, 30, 129.

[9] V. A. Daragan, K. H. Mayo, Prog. Nucl. Magn. Reson. Spectrosc. 1997, 31, 63.

[10] G. Lipari, A. Szabo, J. Am. Chem. Soc. 1982, 104, 4546.

[11] L. E. Kay, D. A. Torchia, A. Bax, Biochemistry 1989, 28, 8972.

[12] A. G. Palmer, M. Rance, P. E. Wright, J. Am. Chem. Soc. 1991, $113,4371$.

[13] K. L. Mayer, M. R. Earley, S. Gupta, K. Pichumani, L. Regan, M. J. Stone, Nat. Struct. Biol. 2003, 10, 962.

[14] A. G. Palmer, Chem. Rev. 2004, 104, 3623.

[15] A. E. Garcia, Phys. Rev. Lett. 1992, 68, 2696.

[16] A. Amadei, A. B. M. Linssen, H. J. C. Berendsen, Proteins Struct. Funct. Genet. 1993, 17, 412.

[17] A. M. Gronenborn, D. R. Filipula, N. Z. Essig, A. Achari, M. Whitlow, P. T. Wingfield, G. M. Clore, Science 1991, 253, 657.

[18] D. Idiyatullin, V. A. Daragan, K. H. Mayo, J. Phys. Chem. B 2003, 107, 2602.

[19] M. Philippopoulos, C. Lim, J. Mol. Biol. 1995, 254, 771.

[20] D. C. Chatfield, A. Szabo, B. R. Brooks, J. Am. Chem. Soc. 1998, $120,5301$.

[21] M. Philippopoulos, A. M. Mandel, A. G. Palmer, C. Lim, Proteins Struct. Funct. Genet. 1997, 28, 481.

[22] D. A. Case, Acc. Chem. Res. 2002, 35, 325.

[23] G. M. Clore, C. D. Schwieters, Biochemistry 2004, 43, 10678.

[24] T. Gallagher, P. Alexander, P. Bryan, G. L. Gilliland, Biochemistry 1994, 33, 4721.

[25] W. L. Jorgensen, D. S. Maxwell, J. Tirado-Rives, J. Am. Chem. Soc. 1996, 118, 11225.

[26] E. Lindahl, B. Hess, D. Van der Spoel, J. Mol. Model. 2001, 7, 306; internet: http://www.gromacs.org.

[27] B. Hess, H. Bekker, H. J. C. Berendsen, J. G. E. M. Fraaije, J. Comput. Chem. 1997, 18, 1463.

[28] S. Miyamoto, P. A. Kollman, J. Comput. Chem. 1992, 13, 952.

[29] T. Darden, D. York, L. Pedersen, J. Chem. Phys. 1993, 98, 10089.

[30] H. J. C. Berendsen, J. P. M. Postma, A. DiNola, J. R. Haak, J. Chem. Phys. 1984, 81, 3684.

[31] A. T. Brünger, P. D. Adams, G. M. Clore, W. L. DeLano, P. Gros, R. W. Grosse-Kunstleve, J. S. Jiang, J. Kuszewski, N. Nilges, N. S. Pannu, R. J. Read, L. M. Rice, T. Simonson, G. L. Warren, Acta Crystallogr. Sect. D 1998, 54, 905.

[32] J. Kuszewski, A. M. Gronenborn, G. M. Clore, J. Am. Chem. Soc. 1999, 121, 2337.

[33] S. Pfeiffer, D. Fushman, D. Cowburn, J. Am. Chem. Soc. 2001, $123,3021$.

[34] O. Lange, H. Grubmüller, unpublished results. 